\title{
Incidence des programmes publics de vaccination contre le rotavirus sur les enfants canadiens
}

\author{
Pia K Muchaal ${ }^{1 \star}$, Matt Hurst ${ }^{1}$, Shalini Desai ${ }^{2}$
}

\section{Résumé}

Contexte : En 2008, le Comité consultatif national de l'immunisation a recommandé la vaccination systématique contre le rotavirus chez les nourrissons canadiens en bonne santé. Au cours des sept années suivantes, huit provinces et deux territoires ont introduit le vaccin contre le rotavirus dans leurs programmes de vaccination financés par le secteur public.

Objectif : Évaluer le fardeau des infections à rotavirus avant et après la mise en œuvre des programmes de vaccination financés par le secteur public.

Méthodes : Nous avons analysé les cas communautaires confirmés en laboratoire de rotavirus signalés au Programme national de surveillance des maladies entériques et les hospitalisations d'enfants de moins de trois ans de 2007 à 2017 avec des codes CIM-10 propres au diagnostic du rotavirus. Les taux de maladie ont été calculés pour chaque province pour les deux années précédant et suivant la mise en œuvre du financement public du vaccin. L'année de mise en œuvre n'a pas été incluse pour tenir compte de la période d'adoption du vaccin. Les taux selon l'âge ont été évalués dans les provinces ou territoires où des données sur cinq ans étaient disponibles l'année suivant le financement public du vaccin. La méthode d'analyse de données avant et après la mise en œuvre et la méthode de " différence dans les différences » ont été appliquées aux données sur les congés hospitaliers afin d'évaluer les changements entre les provinces ou territoires qui financent un programme et ceux qui n'en financent pas.

Résultats : Les cas communautaires d'infection à rotavirus confirmés en laboratoire signalés au Programme national de surveillance des maladies entériques ont diminué de $54 \%$ entre 2010 et 2017. Les taux de congés hospitaliers ont diminué considérablement chez les enfants de six provinces après l'adoption du vaccin contre le rotavirus. Les taux de congés hospitaliers en Alberta, au Manitoba, en Ontario et à l'île-du-Prince-Édouard ont chuté de $53 \%$ à $71 \%$, et de $75 \%$ en Colombie-Britannique et en Saskatchewan.

Conclusion : Le financement public du vaccin contre le rotavirus semble avoir entraîné une réduction importante des cas de rotavirus confirmés en laboratoire qui ont été signalés au Programme national de surveillance des maladies entériques et des taux de congés hospitaliers liés à la gastro-entérite à rotavirus.
Cette oeuvre est mise à la disposition selon les termes de la licence internationale Creative Commons Attribution 4.0

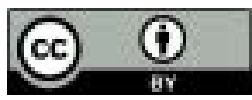

Affiliations

${ }^{1}$ Centre des maladies infectieuses d'origine alimentaire, environnementale et zoonotique, Agence de la santé publique du Canada, Guelph, ON

${ }^{2}$ Centre de l'immunisation et des maladies respiratoires infectieuses, Agence de la santé publique du Canada, Ottawa, ON

\section{*Correspondance :}

pia.muchaal@canada.ca

Citation proposée : Muchaal PK, Hurst M, Desai S. Incidence des programmes publics de vaccination contre le rotavirus sur les enfants canadiens. Relevé des maladies transmissibles au Canada 2021;47(2):108-16.

https://doi.org/10.14745/ccdr.v47i02a02f

Mots-clés : rotavirus, évaluation, vaccination, intervention, fardeau

\section{Introduction}

Le rotavirus est une maladie infectieuse commune transmise d'une personne à une autre par voie fécale-orale. À l'époque précédant la vaccination, la plupart des enfants étaient infectés avant l'âge de cinq ans. En se fondant sur des données limitées disponibles, Thomas et al. ont estimé qu'entre 2000 et 2010, il y a eu une moyenne de 850233 cas de rotavirus communautaire chaque année au Canada (1).
Les manifestations cliniques varient grandement, allant d'une infection asymptomatique à une maladie grave qui peut entraîner une déshydratation grave et la mort. Les enfants immunodéprimés courent un risque accru d'infections graves, prolongées et même de décès par l'infection à rotavirus (2). Chez la plupart des enfants canadiens en bonne santé, l'enfant guérit 
lui-même et la maladie entraîne rarement des séquelles à long terme ou un décès.

Santé Canada a approuvé l'utilisation de deux vaccins, soit RotaTeq (Merck Canada Inc.), un antirotavirus pentavalent à souches réassorties de virus humain-bovin à trois doses (en 2006) (3), et Rotarix (GlaxoSmithKline Inc.), un vaccin monovalent à virus vivant atténué dérivé d'une seule souche humaine à deux doses (en 2007) (4). Le Comité consultatif national de l'immunisation a recommandé l'utilisation de RotaTeq (RV5) en 2008 et de Rotarix en 2010 (5) chez les nourrissons en bonne santé.

Le financement public des vaccins relève des provinces et des territoires du Canada, et les calendriers de vaccination peuvent varier d'une compétence territoriale à l'autre. À la fin de la période couverte par cette évaluation (2010 à 2017), huit provinces et deux territoires avaient inclus la vaccination contre le rotavirus dans leur calendrier de vaccination systématique des nourrissons. De ces dix provinces et territoires, sept sont pris en compte dans la présente étude (figure 1). Les introductions ont été échelonnées de décembre 2010 à décembre 2015. En août 2018, neuf provinces et trois territoires avaient inclus la vaccination contre le rotavirus dans leurs calendriers.

\section{Objectifs}

Notre objectif était d'étudier l'incidence des programmes de vaccination contre le rotavirus financés par l'État en effectuant une analyse nationale du fardeau des infections à rotavirus au Canada avant et après la mise en œuvre des programmes de vaccination financés par l'État et de comparer les provinces et territoires qui ont adopté le vaccin dans leurs calendriers de vaccination de routine respectifs avec ceux qui ne l'ont pas fait.

\section{Méthodes}

\section{Sources de données}

Le Programme national de surveillance des maladies entériques (PNSME) fait le suivi hebdomadaire du nombre de cas confirmés en laboratoire d'infections à rotavirus d'origine communautaire signalés par tous les laboratoires de santé publique provinciaux, à l'exception de ceux du Québec. Toutefois, le rotavirus n'est pas une maladie à déclaration obligatoire à l'échelle nationale au Canada, et les analyses en laboratoire et les rapports varient selon la province ou le territoire. Seule une fraction des cas est déclarée, ce qui entraîne une sous-estimation de l'ampleur de la maladie. De plus, l'information démographique n'est pas disponible pour ces cas. Malgré ces limites, l'ensemble de données du PNSME sert d'indicateur des tendances de l'infection dans la collectivité.

Nous avons obtenu le nombre hebdomadaire de cas de rotavirus signalés au PNSME entre 2007 et 2017 à partir de la base de données du PNSME. Un examen de l'ensemble de données a révélé une rareté de signalements entre 2007 et 2009. Par conséquent, seules les données à partir de 2010 ont été présentées aux fins d'analyse.

À l'aide de la Base de données sur les congés des patients de l'Institut canadien d'information sur la santé, nous avons

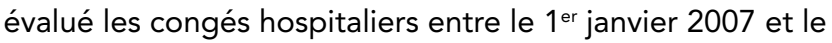
31 décembre 2017. La Base de données sur les congés des patients de l'Institut canadien d'information sur la santé saisit des données administratives, cliniques et démographiques sur tous les congés des soins de courte durée dans toutes les provinces et tous les territoires du Canada, à l'exception du Québec. Les diagnostics cliniques sont classés selon la dixième révision des normes de codage de la Classification statistique internationale des maladies et des problèmes de santé connexes (CIM-10). Nous avons évalué les dossiers individuels des congés de patients chez les enfants de moins de trois ans avec des dates

Figure 1 : Calendrier d'adoption des programmes publics de vaccination contre le rotavirus par les provinces, 2010 à 2015

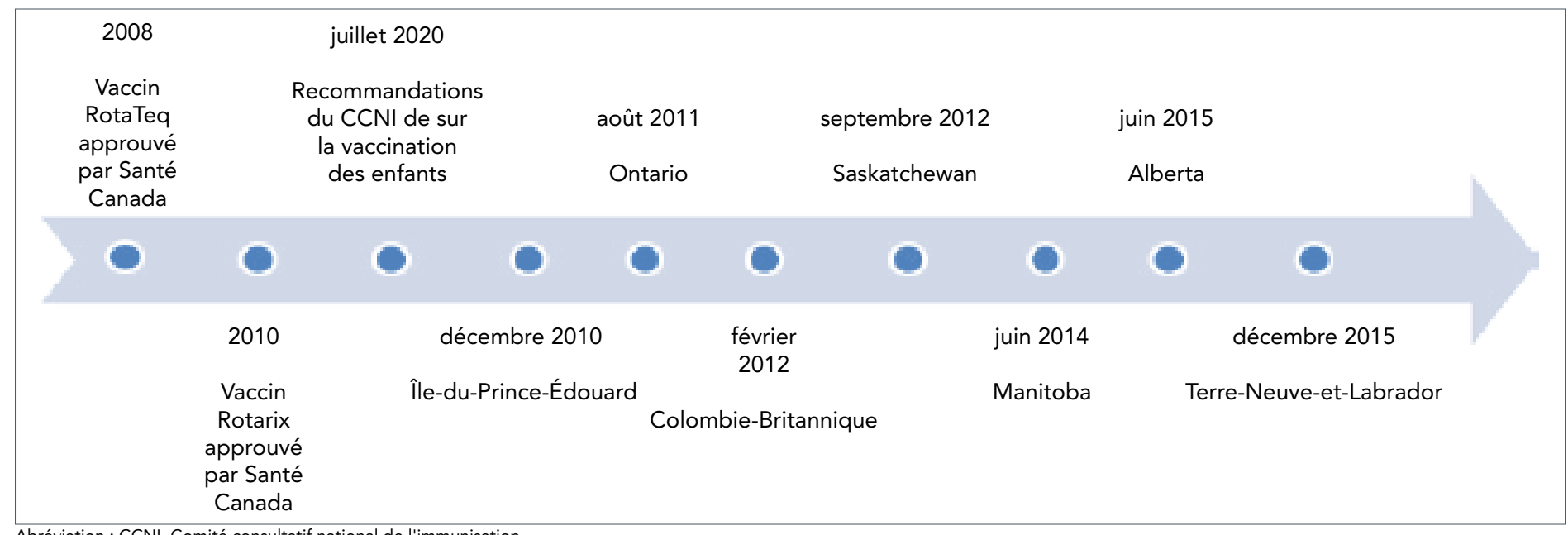


d'admission inscrite dans la Base de données sur les congés des patients de l'Institut canadien d'information sur la santé entre janvier 2007 et décembre 2017. Nous avons défini la mesure du résultat principal comme étant une personne ayant :

- Le code de diagnostic principal de l'entérite aiguë à rotavirus (A080.0)

- Au moins un des diagnostics enregistrés séparément, qui sont de 2 à 16 , d'un code d'entérite aiguë à rotavirus (A080.0)

Le gouvernement de la Nouvelle-Écosse a financé le vaccin contre le rotavirus en 2019. Bien que la date de mise en œuvre se situe en dehors de la période considérée dans cette étude, l'une des autorités sanitaires de la province a participé à un programme de vaccination en décembre 2010 dans le but de comparer l'efficacité de deux systèmes d'administration du vaccin (6). Par conséquent, aux fins de la présente évaluation, la Nouvelle-Écosse est considérée comme une province qui avait également un programme de vaccination.

Les Territoires du Nord-Ouest, le Yukon et le Nunavut ont été exclus de l'analyse en raison d'un manque de rapports provinciaux/territoriaux ou d'un manque de données. Au total, ces trois territoires et le Québec représentent environ $23 \%$ de la population canadienne.

Nous avons défini les périodes avant et après le financement comme les deux années précédentes et les deux années suivant l'année où le vaccin a été adopté dans le calendrier de vaccination. L'année de l'introduction du vaccin a été exclue du calcul du taux pour tenir compte de la période d'adoption du vaccin.

\section{Analyse statistique}

Données du Programme national de surveillance des maladies entériques - infections communautaires à rotavirus

Nous avons résumé les chiffres nationaux des cas de rotavirus signalés au PNSME par les provinces participantes chaque semaine et chaque année afin de refléter les tendances générales des cas dans les collectivités. Le début de la saison du rotavirus a été désigné comme les deux premières semaines consécutives où le nombre de cas hebdomadaires dépassait de $15 \%$ ou plus la valeur médiane annuelle. De même, la fin de la saison était définie comme les deux dernières semaines consécutives où le nombre de cas était de $15 \%$ ou moins de la valeur médiane. La semaine où le nombre de cas déclarés est le plus élevé est appelée la période de pointe de la saison.

Données de la Base de données sur les congés des patients de l'Institut canadien d'information sur la santé hospitalisations

Les enfants de moins de trois ans ont été regroupés dans I'une des catégories d'âge suivantes : moins de 12 mois, 12 à 23 mois et 24 à 35 mois. Les estimations de la population de Statistique Canada ont été utilisées pour calculer les taux annuels selon l'âge de la gastro-entérite à rotavirus (GERV) et pour les périodes de référence (7). Les taux et les intervalles de confiance à $95 \%$ ont été calculés pour estimer la différence entre les périodes d'inclusion avant et après le vaccin à l'aide du progiciel statistique SAS version 9.3 (SAS Institute Inc., Cary, Caroline du Nord, États-Unis). La signification statistique a été définie comme étant $p<0,05$ à partir d'un critère à deux volets de Wald.

\section{Approche de différence dans les différences}

Nous avons utilisé l'approche de "différence dans les différences», une technique appliquée pour évaluer les changements dans les politiques des soins de santé (8), afin d'évaluer l'incidence des vaccins sur les GERV indépendamment des hospitalisations. L'aspect clé de l'analyse de différence dans les différences est qu'en plus d'effectuer une simple comparaison des taux avant et après l'intervention (i.e. le financement public du vaccin) pour voir si les taux ont changé, on effectue également un ajustement pour la situation hypothétique où il n'y aurait pas eu d'intervention et un autre événement aurait pu être responsable du changement observé. Par exemple, une saison moins virulente de rotavirus après la mise en œuvre pourrait donner l'impression que le vaccin est plus efficace qu'il ne l'est en réalité. L'approche de différence dans les différences corrige cette situation.

\section{Résultats}

\section{Rotavirus dans la communauté}

Au total, 5474 cas ont été signalés au PNSME entre 2010 et 2017 , dont $76 \%$ au cours des quatre premières années (2010 à 2013). Des baisses soutenues et importantes $(p<0,05)$ dans les cas annuels déclarés à l'échelle nationale au PNSME se sont produites dans les années subséquentes (2014 à 2017). Pendant la période de pointe de la saison du rotavirus de 2011, 137 cas ont été signalés (médiane annuelle $=14$ par semaine) comparativement à une période de pointe de 15 cas en 2017 (médiane $=6$ ) (tableau 1; figure 2). Au cours de la période de sept ans, la durée de la saison du rotavirus a varié entre 25 et 31 semaines. Entre 2010 et 2015, de $90 \%$ à $94 \%$ des cas annuels ont été signalés pendant la saison du rotavirus. Entre 2016 et 2017, une diminution d'environ 4 \% de cas a été signalée au cours de la saison, et un plus grand nombre de cas sporadiques ont été signalés à d'autres périodes de l'année.

\section{Données de la Base de données sur les congés des patients de l'Institut canadien d'information sur la santé - congés hospitaliers}

Au cours de la période à l'étude (2007 à 2017), les nourrissons et les enfants de moins de trois ans représentaient $70 \%(N=7668)$ de tous les congés hospitaliers pour une GERV. Dans ce groupe d'enfants, la GERV était le « diagnostic principal » pour $82 \%$ ( $n=5379$ ) des congés hospitaliers. Les garçons représentaient $56 \%$ des cas de congés pour les enfants de moins de trois ans. 
Tableau 1 : Cas de rotavirus signalés annuellement au Programme national de surveillance des maladies entériques, 2010 à 2017

\begin{tabular}{|c|c|c|c|c|c|c|c|c|}
\hline Statistiques & 2010 & 2011 & 2012 & 2013 & 2014 & 2015 & 2016 & 2017 \\
\hline $\begin{array}{l}\text { Jombre total de } \\
\text { as déclarés }\end{array}$ & 828 & 1573 & 803 & 773 & 425 & 433 & 258 & 381 \\
\hline $\begin{array}{l}\text { ourcentage du } \\
\text { otal sur } 10 \text { ans } \\
\% \text { ) }\end{array}$ & 15,1 & 28,7 & 14,7 & 14,1 & 7,8 & 7,9 & 4,7 & 7,0 \\
\hline $\begin{array}{l}\text { Jombre médian } \\
\text { le cas }\end{array}$ & 8 & 14 & 9 & 6 & 4 & 5 & 3 & 6 \\
\hline \multicolumn{9}{|c|}{ 'ériode de la saison } \\
\hline $\begin{array}{l}\text { Début (semaine } \\
\text { du PNSME) }\end{array}$ & 1 & 1 & 4 & 3 & 2 & 2 & 4 & 5 \\
\hline $\begin{array}{l}\text { Période de } \\
\text { pointe }\end{array}$ & 15 & 17 & 19 & 16 & 11 & 15 & 22 & 17 \\
\hline Fin & 29 & 28 & 31 & 28 & 33 & 28 & 32 & 31 \\
\hline Durée (semaines) & 29 & 28 & 27 & 25 & 31 & 26 & 28 & 26 \\
\hline $\begin{array}{l}\text { Jombre de } \\
\text { as pendant la } \\
\text { ériode de pointe }\end{array}$ & 65 & 137 & 56 & 70 & 27 & 35 & 18 & 27 \\
\hline $\begin{array}{l}\text { ourcentage } \\
\text { le cas annuels } \\
\text { ignalés pendant } \\
\text { a saison (\%) }\end{array}$ & 91,4 & 94,4 & 90,5 & 91,6 & 92,2 & 90,8 & 86,0 & 86,3 \\
\hline
\end{tabular}

Abréviation : PNSME, Programme national de surveillance des maladies entériques

Dans l'ensemble, les taux canadiens de congés hospitaliers liés au rotavirus ont diminué après le début du financement de la vaccination contre le rotavirus par les provinces à la suite des recommandations du Comité consultatif national de l'immunisation de 2010 (tableau 2). Une comparaison nationale des périodes de financement avant et après la vaccination a montré une réduction de 48,2 cas pour 100000 (IC à $95 \%, 43,9,52,6)$ pendant la période d'observation.
Les taux moyens de congés hospitaliers des enfants pour une GERV ont diminué de $53 \%$ à $76 \%$ dans l'ensemble des provinces après la mise en œuvre de programmes de vaccination financés par l'État dans six des sept provinces, soit l'île-du-Prince-Édouard, l'Ontario, la Colombie-Britannique, la Saskatchewan, le Manitoba et l'Alberta. Les taux de congés pour une GERV ont également diminué en Nouvelle-Écosse. II y a eu une augmentation graduelle, mais non significative, des taux de congés hospitaliers des enfants à Terre-Neuve-et-Labrador (tableau 2).

Dans les cinq ans qui ont suivi le financement du vaccin contre le rotavirus par les premiers adhérents (Île-du-Prince-Édouard, Ontario, Colombie-Britannique et Saskatchewan), les taux de congés hospitaliers pour une GERV ont diminué chez les enfants de moins de trois ans. Dans ces quatre provinces, ces réductions étaient les plus importantes (plus de $85 \%$ ) chez les nourrissons et les enfants d'un an (figure 3). Il y a également eu une diminution statistiquement significative des taux de congés hospitaliers pour les enfants de deux ans en Ontario et en Saskatchewan.

Les programmes d'immunisation financés par le secteur public, mesurés à l'aide d'une approche de différence dans les différences, ont réduit les taux de congés hospitaliers pour une GERV à l'Île-du-Prince-Édouard, en Ontario et en Saskatchewan $(p \leq 0,01)$ (tableau 3). En Saskatchewan, le programme a produit une baisse marquée de 201,4 cas pour 100000 patients (IC à 95 $\%,-258,1,-144,8)$. Pour les enfants de l'île-du-Prince-Édouard, les taux de congés ont diminué de 182,4 cas pour 100000 patients (IC à $95 \%,-308,6,-56,2)$. En Ontario, on a observé une baisse de 63,1 (IC à $95 \%,-108,9,-17,4)$.

Figure 2 : Cas hebdomadaires confirmés en laboratoire signalés au Programme national de surveillance des maladies entériques, 2010 à 2017

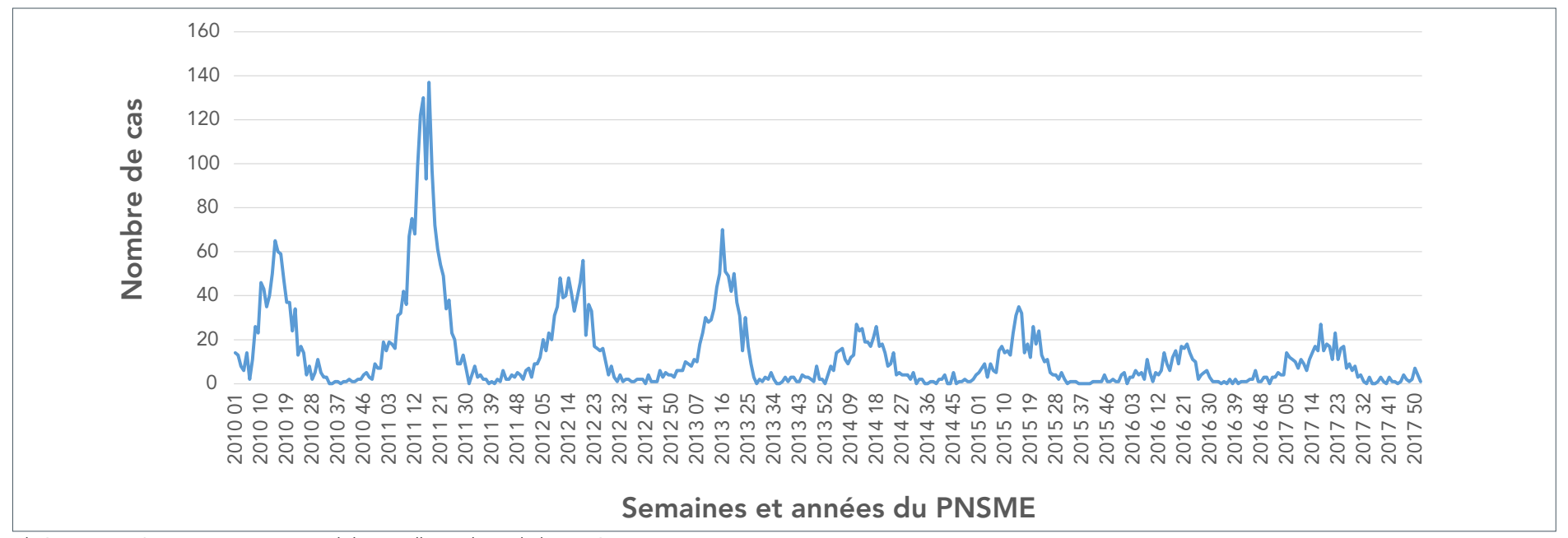

Abréviation : PNSME, Programme national de surveillance des maladies entériques 
Tableau 2 : Taux annuels par 100000 cas et intervalles de confiance à $95 \%$ des congés hospitaliers pour gastro-entérites aiguës à rotavirus chez les enfants de deux ans ou moins

\begin{tabular}{|c|c|c|c|c|c|c|c|c|c|c|}
\hline \multirow{4}{*}{ Province } & \multicolumn{6}{|c|}{ Congés hospitaliers } & \multicolumn{4}{|c|}{$\begin{array}{c}\text { Taux de différence avant et après la } \\
\text { vaccination }\end{array}$} \\
\hline & \multicolumn{3}{|c|}{$\begin{array}{l}\text { Avant le financement de la } \\
\text { vaccination }\end{array}$} & \multicolumn{3}{|c|}{$\begin{array}{l}\text { Après le financement de la } \\
\text { vaccination }\end{array}$} & \multirow{2}{*}{ Taux } & \multirow{2}{*}{$\begin{array}{l}\text { Limite } \\
\text { inférieure }\end{array}$} & \multirow{2}{*}{$\begin{array}{l}\text { Limite } \\
\text { supérieure }\end{array}$} & \multirow{2}{*}{$\begin{array}{l}\% \text { de } \\
\text { variation }\end{array}$} \\
\hline & Taux & $\begin{array}{l}\text { Limite } \\
\text { inférieure }\end{array}$ & $\begin{array}{l}\text { Limite } \\
\text { supérieure }\end{array}$ & Taux & $\begin{array}{l}\text { Limite } \\
\text { inférieure }\end{array}$ & $\begin{array}{l}\text { Limite } \\
\text { supérieure }\end{array}$ & & & & \\
\hline & \multicolumn{3}{|c|}{$2009-2010$} & \multicolumn{3}{|c|}{ 2012-2013 } & \multicolumn{4}{|c|}{ 2009-2010 par rapport à 2012-2013 } \\
\hline $\begin{array}{l}\text { Île-du-Prince- } \\
\text { Édouard }\end{array}$ & 241,7 & 138,3 & 345,0 & 70,8 & 14,1 & 127,5 & $-170,8^{a}$ & $-288,7$ & $-53,0$ & 70,7 \\
\hline Ontario & 96,9 & 90,3 & 103,6 & 45,3 & 40,8 & 49,9 & $-51,6^{\mathrm{a}}$ & $-43,5$ & $-59,6$ & 53,2 \\
\hline \multirow[t]{2}{*}{ Nouveau-Brunswick } & 130,7 & 97,1 & 164,4 & 166,6 & 128,1 & 205,1 & $35,8^{\mathrm{a}}$ & 78,0 & 188,8 & 10,6 \\
\hline & \multicolumn{3}{|c|}{ 2010-2011 } & \multicolumn{3}{|c|}{ 2013-2014 } & \multicolumn{4}{|c|}{ 2010-2011 par rapport à 2013-2014 } \\
\hline $\begin{array}{l}\text { Colombie- } \\
\text { Britannique }\end{array}$ & 31,7 & 24,9 & 38,4 & 7,9 & 4,5 & 11,3 & $-23,8^{a}$ & $-31,4$ & $-16,3$ & 75,1 \\
\hline Saskatchewan & 288,7 & 252,5 & 324,8 & 71,2 & 53,6 & 88,7 & $-217,5^{\mathrm{a}}$ & $-257,7$ & $-177,3$ & 75,3 \\
\hline \multirow[t]{2}{*}{ Nouveau-Brunswick } & 169,6 & 131,2 & 208,0 & 169,8 & 130,6 & 209,7 & 0,23 & $-46,9$ & 47,4 & $\begin{array}{r}\text { Pas } \\
\text { significatif }\end{array}$ \\
\hline & \multicolumn{3}{|c|}{ 2012-2013 } & \multicolumn{3}{|c|}{$2015-2016$} & \multicolumn{4}{|c|}{ 2012-2013 par rapport à 2015-2016 } \\
\hline Manitoba & 14,6 & 6,9 & 22,2 & 4,9 & 0,6 & 9,3 & $-9,6$ & $-18,4$ & $-0,8$ & $\begin{array}{r}\text { Pas } \\
\text { significatif }\end{array}$ \\
\hline \multirow[t]{2}{*}{ Nouveau-Brunswick } & 155,1 & 117,9 & 192,1 & 118,8 & 85,6 & 152,1 & $-36,2$ & $-80,9$ & 8,5 & $\begin{array}{r}\text { Pas } \\
\text { significatif }\end{array}$ \\
\hline & \multicolumn{3}{|c|}{ 2013-2014 } & \multicolumn{3}{|c|}{$2016-2017$} & \multicolumn{4}{|c|}{ 2013-2014 par rapport à 2016-2017 } \\
\hline Alberta & 59,0 & 50,1 & 67,4 & 19,5 & 14,8 & 24,3 & $-39,0^{a}$ & $-96,6$ & $-29,4$ & 67,0 \\
\hline \multirow[t]{2}{*}{ Nouveau-Brunswick } & 169,8 & 130,6 & 209,1 & 121,8 & 88,0 & 155,5 & $-48,1^{a}$ & $-95,1$ & $-1,1$ & 28,3 \\
\hline & \multicolumn{3}{|c|}{ 2014-2015 } & \multicolumn{3}{|c|}{ 2016-2017 } & \multicolumn{4}{|c|}{ 2014-2015 par rapport à 2017} \\
\hline $\begin{array}{l}\text { Terre-Neuve-et- } \\
\text { Labrador }\end{array}$ & 14,6 & 0,3 & 28,9 & 14,8 & $-5,7$ & 35,3 & 0,2 & $-65,3$ & $-16,6$ & $\begin{array}{r}\text { Pas } \\
\text { significatif }\end{array}$ \\
\hline \multirow[t]{2}{*}{ Nouveau-Brunswick } & 143,7 & 107,0 & 180,4 & 122,3 & 74,4 & 170,3 & $-21,3$ & $-68,3$ & 25,5 & $\begin{array}{r}\text { Pas } \\
\text { significatif }\end{array}$ \\
\hline & \multicolumn{3}{|c|}{ 2009-2010 } & \multicolumn{3}{|c|}{ 2012-2013 } & \multicolumn{4}{|c|}{ 2009-2010 par rapport à 2012-2013 } \\
\hline Nouvelle-Écosse & 94,3 & 68,4 & 120,2 & 34,1 & 18,3 & 49,8 & $-60,3^{a}$ & $-119,5$ & $-30,2$ & 64,0 \\
\hline Nouveau-Brunswick & 130,7 & 97,1 & 164,4 & 166,6 & 128,1 & 205,1 & $35,8^{a}$ & 78,0 & 188,8 & 10,6 \\
\hline
\end{tabular}

Remarque : La même période a été utilisée pour évaluer les changements de taux pour la province qui n'applique pas de programme, le Nouveau-Brunswick (nom en caractères gras)

Figure 3 : Congés hospitaliers par 100000 patients pour une gastro-entérite à rotavirus dans les cinq premières provinces ayant adopté le vaccin et au Nouveau-Brunswick, enfants de moins de trois ans, par groupe d'âge
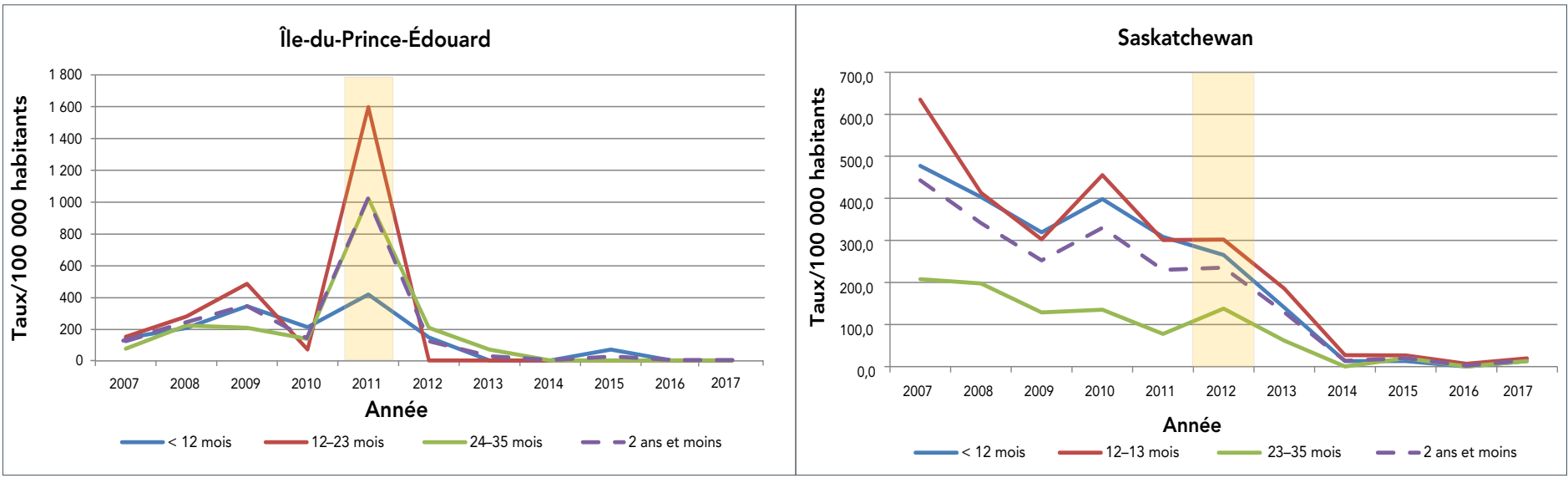
Figure 3 : Congés hospitaliers par 100000 patients pour une gastro-entérite à rotavirus dans les cinq premières provinces ayant adopté le vaccin et au Nouveau-Brunswick, enfants de moins de trois ans, par groupe d'âge (suite)

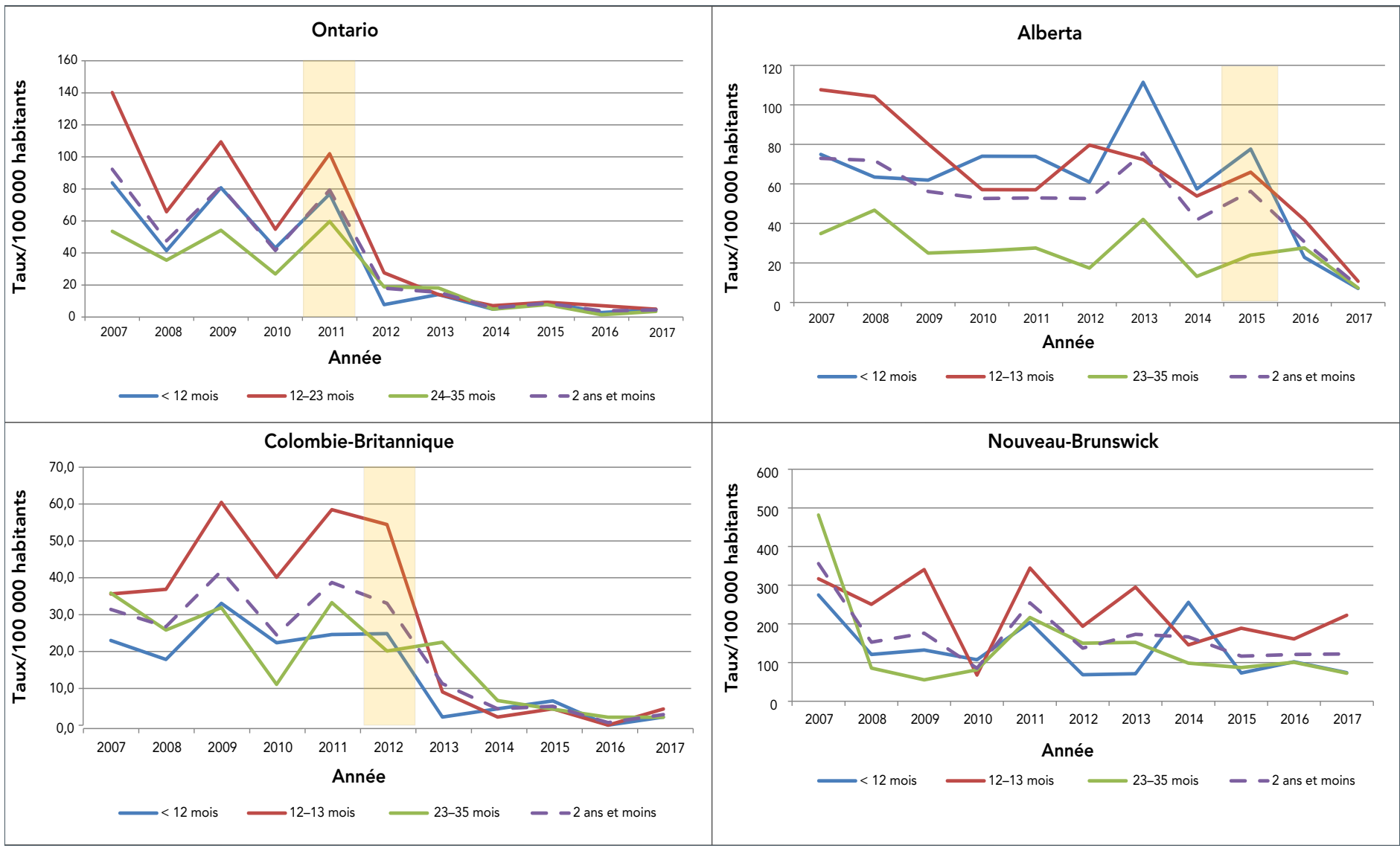

Tableau 3 : Estimations de différence dans les différences : impact des programmes de vaccination financés par le secteur public sur le taux de congés hospitaliers pour une gastro-entérite à rotavirus par 100000 cas, par province

\begin{tabular}{|c|c|c|c|}
\hline \multirow[b]{2}{*}{ Province } & \multicolumn{3}{|c|}{ Effet de l'intervention ${ }^{a}$ entre la province ayant recours au vaccin et la province de référence ${ }^{b}$} \\
\hline & Variation du taux par 100000 cas & $\begin{array}{l}\text { Limite inférieure de } \\
\text { l'intervalle de confiance }\end{array}$ & $\begin{array}{l}\text { Limite supérieure de } \\
\text { l'intervalle de confiance }\end{array}$ \\
\hline Île-du-Prince-Édouard & $-182,4^{c}$ & $-308,6$ & $-56,2$ \\
\hline Ontario & $-63,1^{\mathrm{c}}$ & $-108,9$ & $-17,4$ \\
\hline Colombie-Britannique & $-24,1$ & $-79,5$ & 31,3 \\
\hline Saskatchewan & $-201,4^{c}$ & $-258,1$ & $-144,8$ \\
\hline Manitoba & 3,5 & $-42,9$ & 50,0 \\
\hline Alberta & $-3,2$ & $-51,9$ & 45,5 \\
\hline $\begin{array}{l}\text { Terre-Neuve-et- } \\
\text { Labrador }\end{array}$ & 16,9 & $-46,1$ & 79,8 \\
\hline Nouvelle-Écosse & 9,1 & $-22,4$ & 40,6 \\
\hline
\end{tabular}

a Variation des taux fondée sur les différences dans les différences entre la période avant et après le financement public de l'immunisation

${ }^{b}$ La période de la province de référence (Nouveau-Brunswick) a été appariée à celle de la province qui a recours au vaccin

${ }^{c} p<0,01$ 


\section{Discussion}

Les deux sources de données examinées dans le cadre de cette étude ont permis de relever des taux décroissants de congés hospitaliers et de cas communautaires attribués à des infections à rotavirus dans les régions où le vaccin contre le rotavirus était financé par l'État. Au fil du temps, le financement public semble avoir entraîné des réductions importantes dans i) les rapports de cas de rotavirus confirmés en laboratoire qui ont été signalés par les provinces au PNSME; et ii) les congés hospitaliers liés aux GERV.

Les taux de GERV déclarés au PNSME ont chuté de plus de $50 \%$ au cours des quatre années qui ont suivi le début de la mise en œuvre par les provinces de programmes d'immunisation financés par le secteur public. Les estimations de l'incidence avant et après l'adoption du vaccin sur les taux de congés hospitaliers pour une GERV étaient considérablement plus faibles pour les nourrissons et les enfants d'un an dans les provinces qui ont financé le vaccin entre décembre 2010 et septembre 2012. Les réductions spectaculaires des taux chez les jeunes enfants ne sont pas inattendues, car historiquement, ces groupes d'âge ont les taux de maladie les plus élevés et profiteraient donc le plus de l'effet direct du vaccin avec une bonne couverture dans la population. L'Enquête nationale sur la couverture vaccinale des enfants (9) a indiqué que la couverture du vaccin contre le rotavirus par les provinces qui l'ont adopté dès le début était de $75,4 \%$. Les taux de couverture vaccinale du rotavirus à l'échelle nationale n'étaient pas disponibles avant 2013.

Parmi les provinces qui ont mis en œuvre le vaccin dès le début, la proportion d'enfants de moins de deux ans l'ayant reçu était semblable: En 2015, la Colombie-Britannique a indiqué que $75 \%$ des enfants de moins de deux ans avaient reçu les deux doses recommandées du vaccin contre le rotavirus (10). La même année, 80 \% des enfants de moins de huit mois en Saskatchewan avaient reçu les deux doses recommandées du vaccin contre le rotavirus (11). Les évaluations de la couverture en Ontario ont montré que l'adoption du vaccin contre le rotavirus avait augmenté à 83 \% en 2014 (12). À l'Île-du-Prince-Édouard, où la vaccination des nourrissons est assurée exclusivement par des infirmières de la santé publique, les estimations de couverture sont supérieures à $90 \%$ (5).

Bien qu'elle n'ait pas été aussi importante, on a observé une nette diminution du nombre d'événements associés à la GERV chez les enfants en Alberta. Ces données peuvent indiquer des taux de couverture plus faibles que ceux des provinces qui ont adopté le vaccin dès le début, car les programmes n'étaient en cours que vers la fin de la période d'étude. Les estimations de la méthode de différence dans les différences pour la Colombie-Britannique et l'Alberta n'étaient pas statistiquement significatives, probablement parce que les effets étaient beaucoup plus faibles et que l'effet était donc plus difficile à détecter. L'approche de différence dans les différences n'a pas permis de réduire de façon significative le nombre de congés hospitaliers pour une GERV en Nouvelle-Écosse. Nos résultats s'alignent aux conclusions de l'enquête menée par Sanford et al. (6) qui visait à évaluer l'efficacité relative des infirmières de santé publique et des cabinets de médecins en tant que systèmes de vaccination. Les auteurs ont noté qu'il n'y avait pas de réduction des hospitalisations liées à la GERV dans les régions à l'étude où la couverture vaccinale était faible. La couverture vaccinale était inférieure à $40 \%$ en Nouvelle-Écosse.

Les taux de congés hospitaliers pour une GERV chez les enfants de moins de deux ans ont diminué de plus de $80 \%$ entre les périodes précédant et suivant l'adoption du vaccin. En revanche, les taux de GERV au Nouveau-Brunswick, la province de référence, sont demeurés inchangés. Ces données soutiennent l'affirmation selon laquelle le financement public du vaccin au Canada a été la cause de la diminution des infections à rotavirus et non un troisième facteur temporel, comme une saison plus douce d'infections à rotavirus.

À ce jour, trois provinces canadiennes, soit l'Ontario, l'île-duPrince-Édouard et le Québec, ont réalisé d'importantes études de cohorte ou populationelles de l'impact de la vaccination pour leur province respective. Chacune de ces études a démontré d'importantes réductions de la GERV à la suite de la mise en œuvre de programmes financés par l'État. Le programme en Ontario s'est traduit par une réduction pouvant aller jusqu'à $79 \%$ des hospitalisations de GERV chez les enfants et les jeunes de moins de 20 ans $(12,13)$. Une étude menée au Québec a révélé une réduction de $80,1 \%$ des résultats positifs aux tests de GERV à l'Hôpital de Montréal pour enfants de 2012 à 2013, un an après l'introduction du vaccin, comparativement à 2006 à 2009 (14). À l'Île-du-Prince-Édouard, un programme universel de vaccin contre le rotavirus chez les nourrissons, administré par des infirmières de santé publique, a permis d'éliminer les hospitalisations de GERV chez les enfants de moins de 24 mois à la deuxième année du programme (5).

Les baisses observées dans l'étude nationale présentée ici et les évaluations provinciales individuelles concordent avec les constatations dans plusieurs pays à revenu élevé où les hospitalisations liées aux GERV ont diminué en raison de programmes de vaccination semblables. Les enquêtes sur l'impact des campagnes de vaccination contre le rotavirus menées dans les pays à revenu élevé ont fait état de réductions modestes à spectaculaires des hospitalisations pour le rotavirus, selon la population évaluée $(15,16)$.

La vaccination peut entraîner des changements dans la transmission des maladies, réduisant ainsi les maladies directement dans la population vaccinée et indirectement chez les personnes non vaccinées (17). L'immunité indirecte peut être générée par l'immunité communautaire (18). Les données disponibles pour cette étude sont insuffisantes pour déterminer si les enfants non vaccinés ont été épargnés par la maladie. 
Toutefois, dans le cadre d'un programme de vaccination financé par l'État, on suppose que la majorité de la population cible recevra le vaccin.

\section{Limites}

Notre étude comporte plusieurs limites. Premièrement, nous ne connaissions pas l'état de vaccination des cas. De plus, les infections à rotavirus ne sont pas des maladies déclarables à l'échelle nationale, et il n'y a donc pas de système de surveillance unique pour saisir toutes les occurrences de rotavirus dans les collectivités canadiennes. Nous avons donc utilisé comme substituts un programme national de surveillance et des données sur les congés hospitaliers. Cependant, cette méthode n'a pas été sans difficulté. Les données du PNSME ne contiennent pas d'information sur l'âge et le sexe, et les cas déclarés peuvent être plus âgés que le groupe d'âge considéré dans cette étude. Les tests diagnostiques, limités aux cas symptomatiques, entraînent une sous-représentation des cas signalés dans toutes les bases de données.

De plus, la présentation clinique de la maladie à rotavirus peut être indiscernable parmi autres formes de diarrhée aiguë et la plupart des cas sont traités de façon symptomatique. Le traitement symptomatique des cas de gastro-entérite aiguë et la nature de la déclaration de la GERV entraînent des taux annuels d'infection relativement faibles. Cela crée un problème dans l'interprétation du fardeau de la maladie, car ces taux d'infection sont, dans une large mesure, biaisés négativement. Néanmoins, nous nous sommes surtout intéressés aux tendances, c'est-à-dire à la façon dont ces taux peuvent avoir diminué à la suite des programmes de vaccination. Pour ce faire, nous supposons que tous les biais de déclaration qui existaient dans chaque système demeurent les mêmes d'une année à l'autre.

Il convient également de noter que les données sur les hôpitaux du Québec ne sont pas disponibles dans le système de la Base de données sur les congés des patients de l'Institut canadien d'information sur la santé, et que les données sur les congés hospitaliers des Territoires du Nord-Ouest, du Yukon et du Nunavut sont rares. Ces quatre provinces et territoires, qui représentent environ $23 \%$ de la population canadienne, n'ont pas été incluses dans cette étude.

Bien que nous ayons observé une diminution importante du fardeau de la maladie après l'introduction du vaccin, les réductions ne sont peut-être pas attribuables uniquement au vaccin. Des années de faible activité de GERV ont été signalées en l'absence de vaccination, comme ce fut le cas aux Pays-Bas en 2013 à 2014 (19). Les fluctuations naturelles de la prévalence des maladies auraient pu contribuer aux observations présentées ici. L'utilisation de l'estimateur de différence dans les différences atténue cette limitation.

\section{Conclusion}

En résumé, il s'agit d'une évaluation nationale des programmes de vaccination contre le rotavirus au Canada. Notre évaluation fait appel à une approche novatrice, la méthodologie de différence dans les différences, pour montrer que, selon le moment où les programmes ont été mis en œuvre, les taux d'infections à rotavirus et les hospitalisations ont diminué. II faudra mener d'autres enquêtes à l'aide de méthodes novatrices pour déceler d'autres changements au fil du temps.

\section{Déclaration des auteurs}

P. K. M. - Conceptualisation, recherche, curation de données, analyse formelle, rédaction du manuscrit original

M. H. - Méthodologie, révision et édition du manuscrit

S. D. - Conceptualisation, révision et édition du manuscrit

\section{Intérêts concurrents}

Aucun.

\section{Remerciements}

Ce travail a été appuyé par l'Agence de la santé publique du Canada.

\section{Financement}

Tous les efforts liés à ce projet ont été entrepris dans le cadre des obligations du Centre des maladies infectieuses d'origine alimentaire, environnementale et zoonotique et du Centre de l'immunisation et des maladies respiratoires infectieuses. Aucune source externe de financement n'a été fournie pour l'achèvement de ces travaux.

\section{Références}

1. Thomas MK, Murray R, Flockhart L, Pintar K, Pollari F, Fazil A, Nesbitt A, Marshall B. Estimates of the burden of foodborne illness in Canada for 30 specified pathogens and unspecified agents, circa 2006. Foodborne Pathog Dis 2013;10(7):639-48. DOI PubMed

2. Salvadori M, Le Saux N. Recommendations for the use of rotavirus vaccines in infants. Paediatr Child Health 2010;15(8):519-28. DOI PubMed

3. Santé Canada. Sommaire des motifs de décision. Ottawa (ON) : Santé Canada; (modifié 2019-10-21; accédé 202005-05). https://hpr-rps.hres.ca/reg-content/sommaire-moti f-decision-detailOne.php?linkID=SBD00130 
4. Santé Canada. Sommaire des motifs de décision. Ottawa (ON) : Santé Canada; (modifié 2019-10-21; accédé 202005-05). https://hpr-rps.hres.ca/reg-content/sommaire-moti f-decision-detailOne.php?linkID=SBD00129

5. Une déclaration d'un comité consultatif (DCC) Comité consultatif national de l'immunisation (CCNI). Déclaration sur l'usage recommandé du vaccin antirotavirus pentavalent à souches réassorties de virus humain-bovin. Relevé des maladies transmissibles au Canada 2008;34(DCC-1):1-33. https://www.canada.ca/fr/sante-publique/services/ rapports-publications/releve-maladies-transmissibles-canadarmtc/numero-mensuel/2008-34/declaration-recommand e-vaccin-antirotavirus-pentavalent-souches -reassorties-virus-humain-bovin.html

6. Sanford C, Langley JM, Halperin SA, Zelman M and MURVP Maritime Universal Rotavirus Vaccination Program. A universal infant rotavirus vaccine program in two delivery models: effectiveness and adverse events following immunization. Hum Vaccin Immunother 2015;11(4):870-4. DOI PubMed

7. Statistique Canada. Tableau 17-10-0005-01 Estimations de la population au 1er juillet, par âge et sexe. Ottawa (ON) : Statistique Canada; 2017 (accédé 2020-03-15). https://www150.statcan.gc.ca/t1/tbl1/fr/tv.action?pid=17100 00501\&request_locale $=\mathrm{fr}$

8. Dimick JB, Ryan AM. Methods for evaluating changes in health care policy: the difference-in-differences approach. JAMA 2014;312(22):2401-2. DOI PubMed

9. Statistique Canada. Enquête sur la couverture vaccinale nationale des enfants, 2015. Le Quotidien, 28 juin 2017. https://www.statcan.gc.ca/daily-quotidien/170628/ dq170628a-fra.pdf

10. BC Centre for Disease Control. Immunization uptake in children by the second birthday, 2007-2017. Vancouver (BC): BCCDC; (modifié 2018-01-15; accédé 2018-04-16). http://www.bccdc.ca/Health-Professionals-Site/ Documents/2_Year_Old_Coverage_2005-2015_Birth_ Cohorts\%20(2).pdf

11. CANVAX. Vaccine preventable disease monitoring report: rotavirus, 2015 and 2016. Saskatchewan (SK): Saskatchewan Ministry of Health Population Health Branch; 2017 July (accédé 2018-04-16). https://www.saskatchewan.ca/search\#q =rotavirus\&sort=relevancy
12. Wilson SE, Rosella LC, Wang J, Le Saux N, Crowcroft NS, Harris T, Bolotin S, Deeks SL. Population-level impact of Ontario's infant rotavirus immunization program: evidence of direct and indirect effects. PLoS One 2016;11(5):e0154340. DOI PubMed

13. Wilson SE, Chung $H$, Schwartz KL, Guttmann A, Deeks SL, Kwong JC, Crowcroft NS, Wing L, Tu K. Rotavirus vaccine coverage and factors associated with uptake using linked data: Ontario, Canada. PLoS One 2018;13(2):e0192809. DOI PubMed

14. Comeau JL, Gagneur A, Quach C. Impact of a publicly funded monovalent rotavirus vaccination program in the Province of Quebec (Canada). Vaccine 2016;34(7):893-8. DOI PubMed

15. Panozzo CA, Becker-Dreps S, Pate V, Weber DJ, Jonsson Funk M, Stürmer T, Brookhart MA. Direct, indirect, total, and overall effectiveness of the rotavirus vaccines for the prevention of gastroenteritis hospitalizations in privately insured US children, 2007-2010. Am J Epidemiol 2014;179(7):895-909. DOI PubMed

16. Patel MM, Glass R, Desai R, Tate JE, Parashar UD. Fulfilling the promise of rotavirus vaccines: how far have we come since licensure? Lancet Infect Dis 2012;12(7):561-70. DOI PubMed

17. Zepp F, Heininger U, Mertsola J, Bernatowska E, Guiso N, Roord J, Tozzi AE, Van Damme P. Rationale for pertussis booster vaccination throughout life in Europe. Lancet Infect Dis 2011;11(7):557-70. DOl PubMed

18. Tsai CJ, Griffin MR, Nuorti JP, Grijalva CG. Changing epidemiology of pneumococcal meningitis after the introduction of pneumococcal conjugate vaccine in the United States. Clin Infect Dis 2008;46(11):1664-72. DOI PubMed

19. Hahné $S$, Hooiveld $M$, Vennema $H$, van Ginkel $A$, de Melker $\mathrm{H}$, Wallinga J, van Pelt W, Bruijning-Verhagen P. Exceptionally low rotavirus incidence in the Netherlands in $2013 / 14$ in the absence of rotavirus vaccination. Euro Surveill 2014;19(43):20945. DOI PubMed 